\title{
Finding Chances to Intervene Before the Fatal Overdose: Linking ED and Mortality Data
}

\section{Evan Mobley, Chelsea Fischer, Andrew Hunter}

Bureau of Health Care Analysis and Data Dissemination, Missouri Department of Health and Senior Services, Jefferson City, Missouri, United States

Objective

Link emergency department (ED) with death certificate mortality data in order to examine the prior medical history of opioid overdose victims leading up to their death.

\section{Introduction}

In 2017, 951 Missouri residents died from an opioid overdose - a record number for the state [1]. This continues the trend from 2016, which saw an increase of over 30\% in opioid overdose deaths compared to 2015. The Missouri Department of Health and Senior Services (MDHSS) manages several public health surveillance data sources that can be used to inform about the opioid epidemic. Opioid overdose deaths are identified through death certificates which are collected through the vital records system. MDHSS also manages the Patient Abstract System (PAS), which contains ED and inpatient hospitalization data from approximately 132 non-federal Missouri hospitals. PAS contains about 130 variables, which include demographic data, diagnoses codes, procedures codes, and other visit information. Records can have up to 23 diagnosis fields, which are coded using ICD-10-CM (International Classification of Diseases, Clinically Modified). The first diagnosis field is the primary reason for a visit.

\section{Methods}

Linkage and analysis of the data was performed using SAS Enterprise Guide 6.1. Opioid overdose deaths were identified through ICD-10 analysis looking for drug poisoning underlying cause of death codes and opioid-specific codes found in the multiple cause (contributing cause) of death fields. Table 1, below, summarizes the ICD-10 codes used. Mortality data from the 951 decedents were linked to ED data from 2016 and 2017. Records were linked using multiple passes over the ED records. Records were first linked on social security number. Following this linkage, ED records with no initial match went through a second pass and linked on name and date of birth. Finally, a third pass for records still without a match was conducted using date of birth, census tract, and sex. After these passes, the linkages were reviewed to identify any false positives. The 23 diagnosis fields contained in PAS were analyzed to look for patterns in diagnosis coding. ICD-10- CM codes were too broad so CCS (Clinical Classifications Software) categories were utilized.

\section{Results}

In total, 3,500 ED records were linked to the 951 decedents. After removing false positives, the total number of ED records was 3,357. Approximately 70\% (687) of decedents were linked to at least one ED record. One hundred and eighty-eight visits were due to drug overdose (153 opioid overdoses). The most common primary diagnosis CCS categories (category numbers in parentheses) were: substance-related disorders (661), Spondylosis; intervertebral disc disorders; other back problems (205), abdominal pain (251), and other nervous system disorders (95). Collectively, these four categories represented over $20 \%$ of all primary diagnoses. Across all 23 diagnosis fields there were similar results. The most common CCS categories were as follows: substance-related disorders (661), other aftercare (257), essential hypertension (98), and mood disorders (657). Pie charts (Fig. 1 and 2) below show proportions of CCS categories across all diagnoses fields and primary diagnosis broken into three major categories: pain/injury, substance abuse/mental health, and other. In order to reduce the impact of CCS categories with small numbers, these graphics represent only CCS categories that made up $1 \%$ or more of the total collection of diagnoses codes. Of the 687 decedents that were matched successfully to ED records, $96 \%$ had at least one pain/injury or one substance abuse/mental health ICD-CM code in at least one record, and $68 \%$ had both.

\section{Conclusions}

These findings suggest that many overdose decedents visited the ED in the years prior to death. Many of these visits were not due to an overdose; however, they could be indicative of a problem with opioids (i.e. pain, drug-seeking, substance use-related). ED staff and public health professionals could utilize these opportunities to refer patients to recovery services and recommend they heed caution when using opioids.

SDS Annual Conference Proceedings 2019. This is an Open Access article distributed under the terms of the Creative Commons AttributionNoncommercial 4.0 Unported License (http://creativecommons.org/licenses/by-nc/3.0/), permitting all non-commercial use, distribution, and reproduction in any medium, provided the original work is properly cited. 
ISDS 2019 Conference Abstracts

\section{References}

1. Missouri Department of Health and Senior Services. (2018). Missouri Resident Overdose Deaths by Opioid Type. Retrieved September 27, 2018 from https://health.mo.gov/data/opioids/pdf/opioid-dashboard-slide-9.pdf.

Table 1.

\begin{tabular}{|l|l|}
\hline \multicolumn{2}{|c|}{ ICD-10 Codes Used to Identify Opioid Overdose Deaths } \\
\hline Underlying Cause of Death & Multiple Cause (Contributing) of Death \\
\hline X40 - X44.9 (Accidental) & T40.0 (Opium) \\
\hline X60 - X64.9 (Suicide) & T40.1 (Heroin) \\
\hline \multirow{3}{*}{ X85 - X85.9 (Homicide) } & T40.2 (Other Opioids - e.g. Codeine) \\
\hline Y10 - Y14.9 (Undetermined) & T40.3 (Methadone) \\
\cline { 2 - 2 } & T40.4 (Other synthetic narcotics - e.g. Fentanyl) \\
\cline { 2 - 2 } & T40.6 (Other and unspecified narcotics) \\
\hline
\end{tabular}

Table 2.

\begin{tabular}{|l|l|}
\hline \multicolumn{2}{|c|}{ Select CCS Category ICD-10-CM Codes } \\
\hline Substance-Related Disorders & $\begin{array}{l}\text { F1110 - F1999, F550 - F558, O355XX0 - O99325, P0441 - } \\
\text { P962, T400X1A - T40996S }\end{array}$ \\
\hline $\begin{array}{l}\text { Spondylosis; intervertebral disc disorders; } \\
\text { other back problems }\end{array}$ & M4320-M4327, M436, M4600 - M4989, M5000 - M549, \\
\hline Abdominal Pain & M62830, M961, M9920 - M9979 \\
\hline & R100 - R1033, R10811 - R10829, R1084, R109 \\
\hline & B2701 - B2791, G08, G130-G138, G210 - G219, G360 - \\
Other nervous system disorders & G998, G4720 - G4729, G47411 - G47429, G4763, G500 - \\
\hline $\begin{array}{l}\text { Other after care (includes Other long term } \\
\text { (current drug therapy) and long term use of } \\
\text { opiate) }\end{array}$ & Z86011, Z8661, Z8669, Z982 - 489, R900, R9082, Z462, \\
\hline * This is a summary of codes included in each CCS category. Not every code in the ranges listed above may \\
\hline \multicolumn{2}{|c|}{ be associated with that particular CCS category } \\
\hline
\end{tabular}

Figure 1.

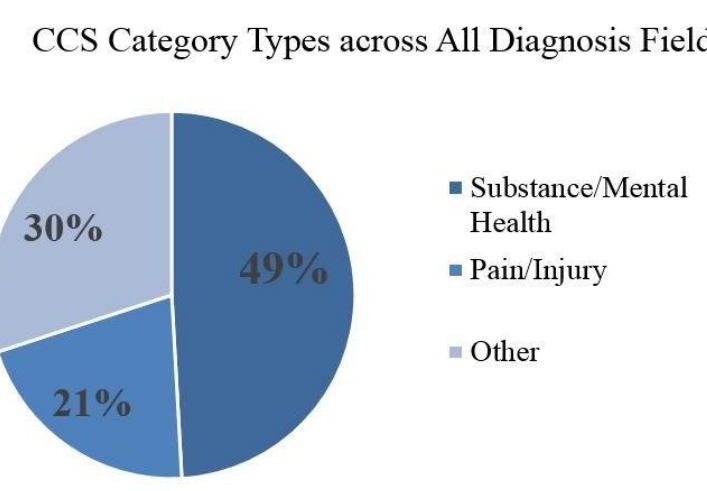

Only CCS categories that made up $1 \%$ or more of the total collection of diagnoses codes are represented in this graphic. The denominator for this data is all captured diagnoses that fell within a CCS category within the $1 \%$ threshold (e.g. one record with 10 complete diagnosis fields would add 10 codes to the denominator, while a record with only 1 field filled would add $1)$. 
Figure 2.

\section{CCS Category Types across Primary Diagnosis Field}

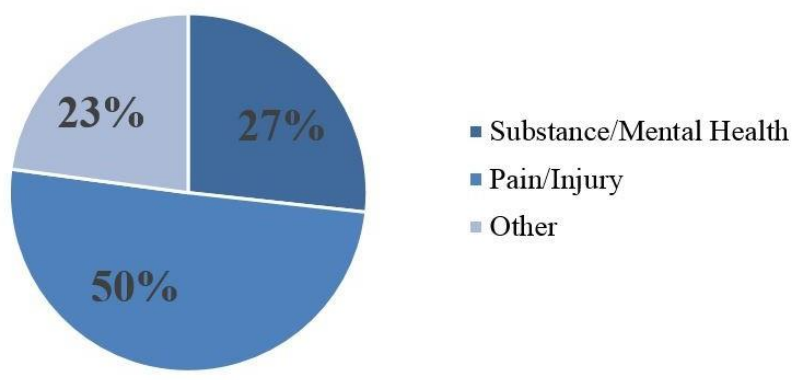

Only CCS categories that made up $1 \%$ or more of the total collection of diagnoses codes are represented in this graphic. The denominator for this data is all primary diagnoses that fell within a CCS category within the $1 \%$ threshold. 